\title{
OMSI (Optically Masked Size Increase): Einstein's Fourth 1907 Miracle, Exposed
}

\author{
Rossler $\mathrm{OE}^{*}$ and Kuske F \\ University of Tuebingen, Germany
}

*Corresponding author: Rossler OE, Faculty of Science, University of Tuebingen, Auf der Morgenstelle 8, 72076 Tuebingen, Germany

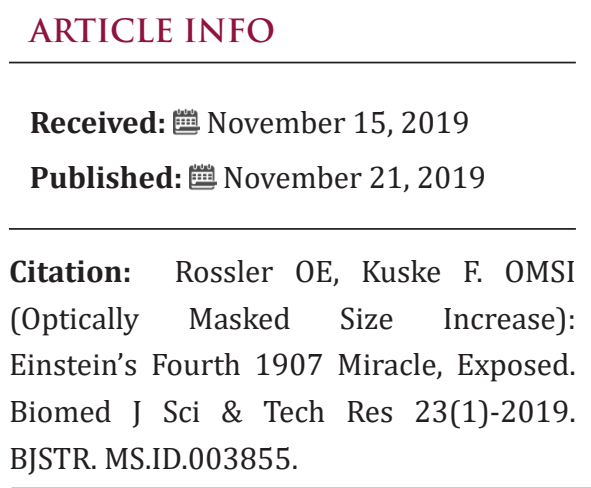

\section{Introduction}

The Einstein equivalence principle of 1907 applies to the interior of a constantly accelerating long Rocketship in free outer space [1]. It is maximally famous because it yielded three radically new correct physical predictions as if plucked from the blue sky (Gravitational redshift; gravitational light curvature; gravitational apparent light slowdown). Now, a fourth implication can be indicated: Horizontal light rays hugging the bottom proceed there in a locally slanted fashion relative to the tip while keeping their horizontal lengths.

This new fact found to be implicit in ref. [1] restores the global speed of light c downstairs in gravitation. Einstein, who reluctantly gave up on this necessity for good 3 years later, would rejoice on learning about the above 4th revolutionary implication of his superhuman brainchild. It is named OMSI here. OMSI rehabilitates Zwicky 1929 ("no cosmic expansion" [2]). Much more incisive, OMSI implies that CERN is unsafe for earth. For CERN is since early 2008 busy betting earth on Hawking radiation, even though Hawking radiation [3] is based on c-nonglobal and hence is nonexistent. CERN for this reason is unable to renew its latest planetary safety report LSAG from early 2008 [4]. However, instead of stopping short CERN has chosen to continue by camouflaging its inability to renew the LSAG by keeping the latter's last version from early 2008 undated so it looks recent.

\section{Conclusion}

To conclude, the OMSI-based fourth implication of Einstein's "happiest thought" from 1907-the optically masked size increase downstairs in gravitation demonstrated above - radically alters humankind's ideas about the cosmos and how to survive in it.

We thank Dieter Fröhlich and the late Klaus Sonnleitner for discussions. For J.O.R.

\section{Acknowledgement}

None.

\section{Conflict of Interest}

No conflict of interest.

\section{References}

1. Einstein A (1907) Über das Relativitätsprinzip und die aus demselben gezogenen Folgerungen. Jahrbuch der Radioaktivität 4: 411-462.

2. Zwicky F (1929) On the red shift of spectral lines through interstellar space. Proceedings of the National Academy of Sciences 15: 773-779.

3. Hawking SW (1975) Particle creation by black holes, Commun Math Phys 43: 199-220.

4. The LSAG Report. 
ISSN: 2574-1241

DOI: $10.26717 /$ BJSTR.2019.23.003855

Rossler OE. Biomed J Sci \& Tech Res

(c) (P) This work is licensed under Creative BY Commons Attribution 4.0 License

Submission Link: https://biomedres.us/submit-manuscript.php

BIOMEDICAL
RESEARCHES $\quad \begin{aligned} & \text { Assets of Publishing with us } \\ & \text { - Global archiving of articles }\end{aligned}$

